\title{
Mapping HIV progression and Co-Factors affecting AIDS survival time in women and men on ART in India
}

\begin{abstract}
HIV is continuum of progressive damage traversed through survival time. Using data from 204 positive women and men, we carried-out survival analysis using Kaplan-Meier curves, Cox proportional hazard regression and Markov-chain model to map CD4 progression and health-condition outcomes. Progression was better among non-defaulters \{adjusted hazard ratio [AHR]-0.11, 95\% CI 0.03-0.31 (Log rank p<0.001) $\left.\left(\mathrm{chi}^{2}=73.62, \mathrm{p}<0.001\right)\right\}$, those with higher self-perceived adherence and early initiation of ART. TB-HIV co-infection translated in lower progression and even lower among women [AHR]-0.08, 95\% CI 0.02$0.28 ; \log$ rank $\mathrm{p}<0.001)\left(\mathrm{chi}^{2}=71.72, \mathrm{p}<0.001\right)$. Similar result was observed in women who reported side effects and who defaulted treatment. Immune reconstitution emerged strongly from Markov-chain model, with striking gender gap. Probability of women maintaining working condition was quite low (0.88) as compared to men (0.94). Transition to better health from bed-ridden state was also lower among women. A health-care delivery genderresponsive.
\end{abstract}

Keywords: HIV, ART, Clinical progression, CD4, Survival
Volume 5 Issue 6 - 2017

\section{S K Singh, Nidhi Sharma}

International institute for Population Sciences, India

Correspondence: SK Singh, International institute for Population Sciences, Govandi Station road, Deonar,Mumbai-400088, India, Email sksingh 1992@yahoo.co.in

Received: December 28, 2016 | Published: August 01, 2017

\section{Introduction}

HIV disease is a continuum of progressive damage to the immune system from the time of infection to the manifestation of severe immunologic damage by opportunistic infections (OI), or low CD4 count that defines AIDS. The time it takes to traverse this spectrum varies greatly from person to person. The period from an AIDS diagnosis to death can be termed as AIDS survival time. The literature of the epidemiology of HIV disease progression has attempted to characterize the distribution of possible lengths of AIDS survival period, to identify tests useful for prognosis and treatment decisions, and to determine what cofactors accelerate or retard the rate of disease progression by affecting the number of CD4 cells in the body. Globally, it is suggest starting treatment when CD4 count falls to350 cells $/ \mathrm{mm}^{3}$ or below. This is because opportunistic infections typically begin to affect people whose CD4 counts are below that level. This is why a CD4 count is often used to determine the stages of HIV disease. Recent research has indicated that it may be easier to maintain higher CD4 counts if started HIV treatment before CD4 counts drop below 350 cells $/ \mathrm{mm}^{3}$. Numerous studies have demonstrated that the baseline CD4 count serves as a significant prognostic indicator for treatment outcome. In one study, patients starting therapy with a CD4 count below 200 cells $/ \mathrm{mm}^{3}$ were almost twice as likely (HR: 1.90) to fail treatment, compared with those starting with a CD4 count higher than 200 cells $/ \mathrm{mm}^{3}$. Another study showed an inverse relationship between the CD4 count at baseline and a risk of progression to AIDS or death. This effect was quite dramatic: the adjusted HR for progression to AIDS or death was 0.24 (95\% CI: $0.20-0.30)$ for patients starting ART with a baseline CD4 count of 200-350 cells $/ \mathrm{mm}^{3}$, compared with patients with a CD4 count below 50 cells $/ \mathrm{mm}^{3}$. Observations on the natural history of HIV infection may no longer hold in an era of more effective antiretroviral therapy. The effectiveness of combination antiretroviral therapy that includes a protease inhibitor in slowing HIV disease progression appears to be altering the disease course significantly. There has been a lot of research about whether very early initiation of therapy could control and break the transmission routes of HIV. The strengthening the initial immune response with early treatment to control infection indefinitely holds out more promise, although it may depend on whether the immune system can control HIV even if therapy is withdrawn at some point, thus making the compliance in ART very critical. Opportunistic infections that occur in the course of increasing HIV immune suppression are largely caused by infectious agents. There is evidence that OI may affect subsequent HIV disease, perhaps by increasing HIV replication during the period of acute disease or by increasing cytokines that in turn impact on disease progression.

\section{Methods and techniques}

\section{Data}

The basic data used in this study have been collected from a mixed method approach. For each PLHIV the qualitative data was collected from two sources: the CD4 record from the ART record Book of the PLHIV and behavioral data collected through survey. The target populations for cross-sectional study were the persons registered at least before Jan 2009 currently visiting ART centers and age group more than 18 years. A total of 204 interviews, with 116 women and 88 men, were completed from Mumbai and Pune city. Kaplan-Meier curves were fitted to assess the change in CD4 (which is considered as probability of death) stratified by CD4 cell count at initiation of treatment for both women and men. Log rank test was used to examine statistical difference between the groups with in both the genders. Time was measured from the start of ART and ended at the date of last follow up or last follow-up for which CD4 count is available. Cox proportional hazard regression model-Hazard ratio (HR) for the timeto-endpoint (last follow up) was estimated using Cox proportional hazard regression model. Factors included in the model were age, default behavior, self-perceived adherence, Tuberculosis and baseline CD4 count as categorical variables. We have used Markov-chain model to study the transition probabilities from one state of heath to another by condensing the reported health condition at the initiation and at the last follow-up. Some of the operational definitions used in the chapter are CD4 $>50 \mathrm{Cells} / \mathrm{mm}^{3}$ was used as the reference 
category. Cell progression is defined as the changes in CD4 cell count with time since treatment initiation. Failure is defined as defaulters/ adherence level/TB-HIV co-infection/WHO stage at initiation. The stratification of the sample was done baseline CD4 count- baseline CD4 count $<=50$ cells $/ \mathrm{mm}^{3}$. Finally the Start point is this analysis is initiation of treatment and Endpoint is the last follow up record.

\section{Results}

The group that we studied in our study, majority was in the age category of 25 years to 44 years. Nearly half of the women and three-fourths of men in our sample reported to be currently married. Level of partner concordance/discordance was assessed among the couple who reported to currently having a regular partner. It is seen that significantly higher proportion of men as compared to women are in discordant couple (44\% men as compared to only $9 \%$ women), whereas, 85 percent women reported to be in concordant couple. It is important to highlight that nearly two-fifths of currently married women and men reported to have remarried through positive matrimony initiated by the NNP+. Hence for this sub-group of respondents the current regular partner was not the partner when they got infected with HIV. Further, 31 percent women as compared to 11 percent men reported to have at least one positive child (Table 1). More positive women than positive men reported higher occurrence and number of side effects. Most commonly reported side effect reported included vomiting, nausea and drowsiness. Side effects are found to be higher among those whose baseline CD4 count was less than 50, whose WHO clinical stage at initiation was 1 and 2, who have more than 2 co-morbidities. Higher ages perceived negative effect of ART, low knowledge of HIV and regimen other than ZLN are the factors significantly affecting occurrence of side effect. We have found that more women suffer from side effects. This may not make them equally vulnerable to higher perceived negative effect but same is not true, despite high side effects, only 17 percent women perceived negative effects of ART where as one fourth of men did perceive a negative effect of ART on their health.Opportunistic infections, which essentially are the signs of weakening immune system was quite prevalent in this group with three out of four respondents ever suffering from any OI. TB one of the most commonly prevalent OIs among PLHIV was the most common presenting illness among people living with HIV adding on to the exiting evidences. The prevalence of OIs was reported to be significantly higher among men as compared to women. This can be attributed to the fact that men show higher risk in terms of many behavioral indicators. The self-perceived adherence was observed high with no profound gender difference. The self-perceived negative effects of ART on health has emerged as the single most important factor contributing to the SPA both for positive women and men. The most important barriers to adherence reported were stigma and discrimination related to disclosure, lack of support, poverty and nutrition, self-assessment of health status, perceived efficacy of treatment, disruption in schedules. Most common conducive factors were improvement in health, counselling and knowledge, financial support, self-efficacy of treatment and perceived health benefits. Gender issues related to adherence identified are dual stigma, lack of financial resources, travel constraint to ART center, economic burden due to partner's status, fear of violence and coercion. Default is marginally higher (although not statistically significant) among women (12.1 percent) as compared to men (11.4 percent). Living arrangement, area of living, age at first sex, and change in occupation due to HIV, treatment interruption, pill burden, TB-HIV co-infection, presence of more than two co-morbidities, negative perception about ART and self-perceived adherence have shown significant association with default behaviour among women. On the other hand, marital status, current occupation, mobility, mode of transmission, treatment interruption, presence of more than two co-morbidities, incidence of any STI in the last one year, treatment from other health care provider, disclosure status, reason for testing and HIV knowledge are significantly associated with default among men. The most common reasons for default were long waiting time at ART centers followed by side effects of treatment, continuation of OI treatment and inability to visit ART centers due to weakness.

Table I Percent Distribution of Positive Women and Men by Positive Partner and Children

\begin{tabular}{|c|c|c|c|c|}
\hline \multirow{2}{*}{ Positive households } & \multicolumn{2}{|c|}{ Women Men } & \multirow{2}{*}{\multicolumn{2}{|c|}{$\begin{array}{l}\text { Total } \\
\%(\mathbf{N})\end{array} \mathbf{X}^{2}$}} \\
\hline & $\%(\mathbf{N})$ & $\%(\mathbf{N})$ & & \\
\hline & $9.3(9)$ & $44.4(32)$ & (4I) & \\
\hline les & (82) & 54.2 & & \\
\hline & & & & \\
\hline itive chilc & $31.2(30)$ & $10.9(7)$ & $23.1(37)$ & $8.9 * * *$ \\
\hline
\end{tabular}

\section{CD4 progression and treatment outcomes}

\section{CD4 progression among positive men and women not reporting default}

We analyzed the CD4 progression in the group of men and women who did not report any default, thus were complying with regimen as prescribed. Below are the graphs showing the progression in terms of CD4 count. Surprisingly, it is seen that improvement in the number of CD4 cell was faster among the patients who started ART after reaching CD4 level 50 Cells $/ \mathrm{cmm}^{3}$ or below. Similar trend was also observed among positive women. This can be attributed to the fact the cases initiating ART below 50 are at a critical health condition and are kept under prophylaxis that instantly regains immune system. Thus reinforcing the importance of compliance. Results from KaplanMeier clearly show a progressive lowering of risk of progression with increasing baseline CD4 cell count among these treatment outcome groups. The results from Cox-proportional hazard model for this group gives an adjusted hazard ratio [AHR] $0.11,95 \%$ CI $0.03-0.31$ $(\log$ rank $\mathrm{p}<0.001)(\mathrm{chi} 2=73.62, \mathrm{p}<0.001)$, for progression in CD4 count among women as compared to men, which means that the chances of CD4 cell progression are lower among defaulter women (hazard ratio $=0.11$ ) compared to defaulter men (Figure 1).

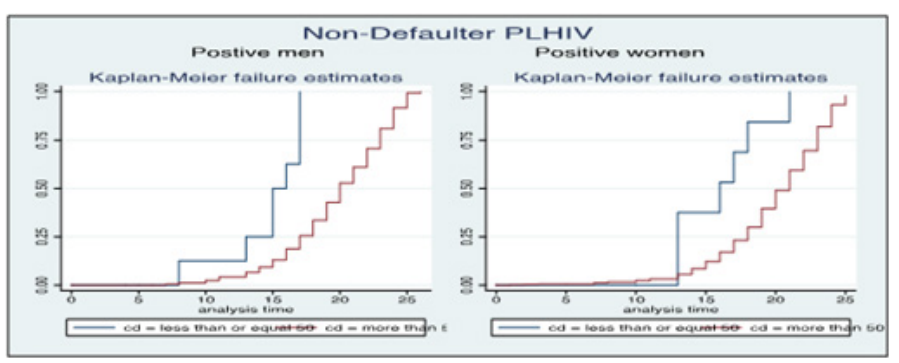

Figure I CD4 progression among Non-Defaulter PLHIVs.

\section{CD4 progression among positive men and women by level of adherence}

The results from the Kaplan-Meier analysis for PLHIV with respect to their SPA shows better probability of attaining higher CD4 among men starting at higher CD4. Similar result was also seen for women. The adjusted hazard ratio shows lower probability of progression among women as compared to men with higher SPA $(\mathrm{AHR}=0.11,95 \%$ CI $0.03-0.36 ; \log$ rank $\mathrm{p}<0.001)(\operatorname{chi} 2=182.31$, $\mathrm{p}<0.001$ ) (Figure 2). 


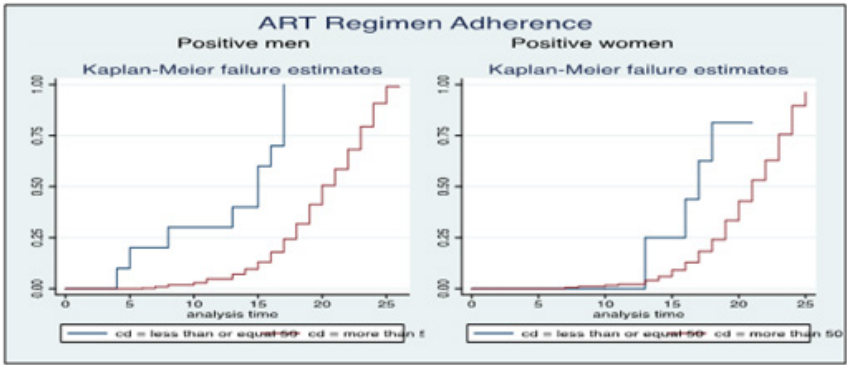

Figure 2 Adherence among male and female patients.

\section{CD4 progression among positive men and women by side effects}

The Kaplan-Meier graphs for PLHIV reporting side effects shows that women shows that women initiating ART at CD4 count $<50$ cells/ $\mathrm{mm}^{3}$ are having high probability of progression of CD4 as compared to men of the same group. Progression among men and women who started ART at later stages seems similar. The results from adjusted hazard ratio shows that as compared to men, women who have side effects have lower probability of CD4 progression (AHR $=0.07,95 \%$ CI 0.03-0.17; Log rank $\mathrm{p}<0.001)\left(\mathrm{chi}^{2}=65.07, \mathrm{p}<0.001\right)$. (Figure 3). The graphs depicting cell progression among PLHIV who have ever had TB-HIV co-infection shows that the probability of progression is low and is even lower among women. The progression for those who started ART at later stages, men are performing better compared to women who stated late. The adjusted hazard ratio shows that those women who reported TB have lower probability of cell progression [AHR] 0.08, 95\% CI 0.02-0.28; Log rank $\mathrm{p}<0.001)\left(\mathrm{chi}^{2}=71.72\right.$, $\mathrm{p}<0.001$ ) (Figure 4), (Table $2 \& 3$ ).

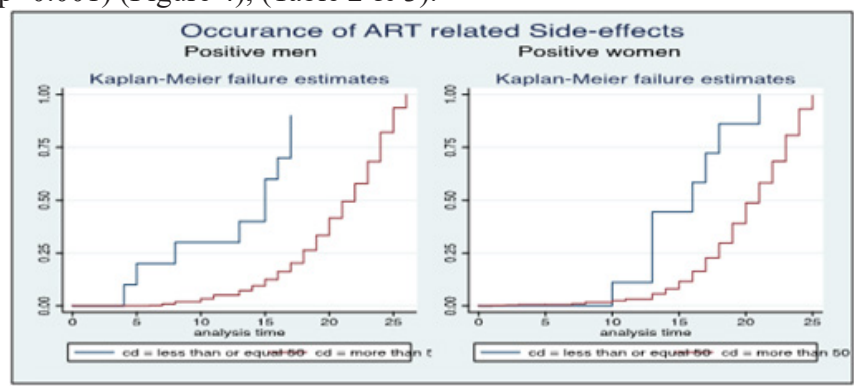

Figure 3 Side effects among male and female patients.

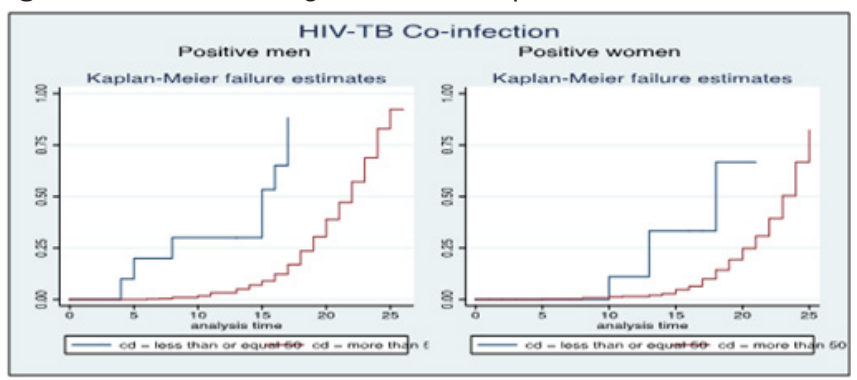

Figure 4 TB-HIV Co-infection among male and female patients.

Table 2 Cox proportional hazard ratio among PLHIV in selected treatment groups

\begin{tabular}{lll}
\hline Adjusted hazard ratio & & Cl \\
\hline Non-Defaulter & $0.1 \mathrm{I}$ & $0.03-0.3 \mathrm{I}$ \\
Adherence & $0.1 \mathrm{I}$ & $0.03-0.36$ \\
Side Effects & 0.07 & $0.03-0.17$ \\
TB-HIV Co-infection & 0.08 & $0.02-0.28$ \\
\hline
\end{tabular}

Table 3 Log-rank test among the PLHIV in selected treatment groups

\begin{tabular}{lll}
\hline Chi-Square & & P value \\
\hline Non-Defaulter & 73.62 & 0.001 \\
Adherence & 182.31 & 0.001 \\
Side Effects & 65.07 & 0.001 \\
TB-HIV Co-infection & 71.72 & 0.001 \\
\hline
\end{tabular}

\section{Health condition transition}

Improvement of health status as part of treatment outcome was assessed in term of the Health condition transition from treatment initiation till last follow-up was also assessed in the study population. It is observed that, nearly 38 percent PLHIV reported that they were seriously ill and were in bed-ridden condition, whereas, 41 percent said they were in ambulatory state and 20.1 percent said they were in working state. After receiving treatment there was a considerable improvement reported in the state of health as 80 percent report to be in working health condition, 19 percent said ambulatory and only one percent reported to be bed-ridden. A comparison between the stages, 'now' and 'before', reveals that among those PLHIV who had been at the bed-ridden stage at the initiation, 78 percent among them reported to be in working condition currently and 23 percent reported ambulatory at last visit to ART center. Both reported an improvement from their initiation stage. We have used Markov-chain model to study the transition probabilities from one state of heath to another by condensing the reported health condition at the initiation and at the last follow-up. The transitional probability in case of both the genders depicts a positive impact of ART on the health condition of the PLHIVs. The results from the model show that transitional probability of moving from bed ridden to working was seen to be 0.75 . Post initiation of ART, the PLHIV who were bed ridden or in ambulatory conditions are most likely to transit in a working condition. The probability of women maintaining the health in a working condition is quite low (transitional probability $=0.88$ ) as compared to men (transitional probability $=0.94$ ), posing a question on the quality of care PLHIV women receive. Similarly, the improvement in health condition among those who were bed-ridden is also much lower for women PLHV as against their male counterparts (Table 4-7).

Table 4 Changes in health condition due to ART

\begin{tabular}{lllll}
\hline $\begin{array}{l}\text { Health Condition } \\
\text { at Time of } \\
\text { Registration }\end{array}$ & $\begin{array}{l}\text { Health Condition During Last Visit to } \\
\text { ART Centre }\end{array}$ & \multirow{2}{*}{ Total } \\
\cline { 2 - 4 } Working & Ambulatory & Bed Ridden & \\
\hline \multirow{2}{*}{ Working } & 37 & 4 & 0 & 41 \\
Ambulatory & 90.2 & 9.8 & 0 & \\
& 68 & 16 & 0 & 84 \\
Bed Ridden & 81 & 19 & 0 & 79 \\
& 59 & 18 & 2 & \\
Total & 74.7 & 22.8 & 2.5 & 204 \\
\hline
\end{tabular}

Table 5 Probability of transition in health condition of PLHIV using Markovchain model

\begin{tabular}{llll}
\hline \multirow{2}{*}{$\begin{array}{l}\text { Condition at } \\
\text { Treatment Initiation }\end{array}$} & \multicolumn{3}{l}{$\begin{array}{l}\text { Condition During The Last Follow-up } \\
\text { Visit }\end{array}$} \\
\cline { 2 - 4 } & Working & Ambulatory Bed Ridden \\
\hline Working & 0.9 & 0.1 & 0 \\
Ambulatory & $0.8 \mathrm{I}$ & 0.19 & 0 \\
Bed ridden & 0.75 & 0.23 & 0.03 \\
$\mathrm{P}_{\mathrm{i}}$ & 0.2 & $0.4 \mathrm{I}$ & 0.39 \\
$\mathrm{P}_{\mathrm{j}}$ & 0.8 & 0.19 & 0.01 \\
\hline
\end{tabular}


Table 6 Probability of transition in health condition of female PLHIV using Markov-chain model

\begin{tabular}{llll}
\hline $\begin{array}{l}\text { Condition at } \\
\text { Treatment Initiation }\end{array}$ & \multicolumn{2}{l}{ Condition During the Last Follow-up Visit } \\
\cline { 2 - 4 } & Working & Ambulatory & Bed Ridden \\
\hline Working & 0.88 & 0.13 & 0 \\
Ambulatory & 0.87 & 0.13 & 0 \\
Bed ridden & 0.71 & 0.24 & 0.05 \\
$P_{i}$ & 0.21 & 0.47 & 0.33 \\
$P_{j c}$ & 0.82 & 0.16 & 0.02 \\
\hline
\end{tabular}

Table 7 Probability of transition in health condition of male PLHIV using Markov-chain model

\begin{tabular}{llll}
\hline Condition at & \multicolumn{3}{l}{ Condition During the Last Follow-up Visit } \\
\cline { 2 - 4 } Treatment Initiation & Working & Ambulatory & Bed Ridden \\
\hline Working & 0.94 & 0.06 & 0 \\
Ambulatory & 0.7 & 0.3 & 0 \\
Bed ridden & 0.78 & 0.22 & 0 \\
$\mathrm{P}_{\mathrm{i}}$ & 0.19 & 0.34 & 0.47 \\
$\mathrm{P}_{\mathrm{ic}}$ & 0.78 & 0.22 & 0 \\
\hline
\end{tabular}

\section{Discussion}

Longitudinal studies of HIV-infected persons have shown that nearly all infected persons have a CD4 lymphocyte count below the mean for seronegative persons and show a progressive loss of these cells over time Geodert et al. ${ }^{1}$ Moss et al. ${ }^{2}$ Phillips et al. ${ }^{3-5}$ Other observations made in the era prior to the advent of combination therapy also suggest it is likely that nearly all HIV-infected persons will eventually lose CD4 lymphocytes and progress to AIDS in the absence of effective treatment. For example, in about 10 years of follow-up of 288 men HIV-seropositive at baseline in the San Francisco General Hospital Study (1983-1993), only one (0.3\%) maintained a CD4 lymphocyte count above 700/ $\mu$ l throughout follow-up and nearly all showed some worsening of other laboratory values predictive of AIDS Cao et al. ${ }^{6}$ Pantaleo et al. ${ }^{6}$ Moss et al. ${ }^{2}$ Buchbinder et al. ${ }^{7}$ AIDSdefining diagnoses have a wide range of average survival times. In the Multicenter Hemophilia Cohort Study median survival after a single AIDS-defining condition ranged from 3 to 51 months for the 10 most common conditions Gali et al. ${ }^{8}$ Ranged from 3 to 51 months for the 10 most common conditions Gali et al. ${ }^{8}$ Endogenous biologic or psycho logic factors, other infections, behaviors, or other environmental factors that alter the natural history of HIV infection may be cofactors for disease progression. Understanding the cofactors for HIV disease may shed light on the pathogenesis of HIV as well as on identifying potential interventions that could slow disease progression. Many potential cofactors for HIV have been investigated. Early initiation of ART has emerged as an important factor for progression as there was a better probability of attaining higher CD4 among men in your study who started ART at higher CD4. There is evidence that OI may affect subsequent HIV disease, perhaps by increasing HIV replication during the period of acute disease or by increasing cytokines that in turn impact on disease progression. Saravolatz et al. ${ }^{9}$ Observed that in a large sample of 5,204 HIV-infected patients mortality rates were higher in all CD4 strata for patients with history of OI prior to entry in the study Saravolatz et al. ${ }^{9}$ CD4 cell progression among PLHIV who have ever had TB-HIV co-infection shows that the probability of progression is low and is even lower among women. Transitional probability of moving from bed ridden to working was more positive in men, highlighting a role gender may be playing in this equation. Post initiation of ART, the PLHIV who were bed ridden or in ambulatory conditions are most likely to transit in a working condition. ${ }^{10}$
Overall a lower progression of CD4 was observed among women as compared to men, irrespective of treatment outcome group. Progressive lowering of risk of progression with increasing baseline CD4 cell count was observed among all treatment outcome groups. The result from the AHR also shows a lower incidence of improvement in CD4 count for women as compared to men implying that even when on similar treatment regimen and suffering from similar conditions, men regain the immune constitution better as compared to women PLHIV. These findings are adding to the findings from the health condition transitional probability where probability that a woman regains a working health condition is much lower than a man's probability of attaining a working heath state. The benefit of ART in terms of immune reconstitution was clear from health condition transition from treatment initiation till last follow-up shown by PLHIVs. It showed a marked improvement with the probability of moving from bed ridden to working to be 0.75 . Surprisingly, gender played a sticking role here creating a wide gap between well-being of women and men. The probability of transition to better health condition was much lower in women as compared to men.

\section{Conclusion and recommendations}

The benefit of ART in terms of immune reconstitution was clear from health condition transition from treatment initiation till last follow-up shown by PLHIVs. Surprisingly, gender played a sticking role here creating a wide gap between well being of women and men. The probability of transition to better health condition was much lower in women as compared to men. Findings from this study raises serious questions on the lack of care the women get to improve her health condition. In the light of these findings, it can be argued that CD4 progression as well as the health condition transitional probability indicates that immune reconstitution and regains of a working health condition is much lower among women than a man. Women have also reported to neglect their health and discontinue treatment due to additional responsibility of care and provide that they are burdened with due to partner's illness. This not only raises a question on quality of care to women, but also underlines the need to have support programmes sensitive to the needs of women.

\section{Acknowledgments}

None.

\section{Conflicts of interest}

None.

\section{References}

1. Goedert JJ, Kessler CM, Aledort LM, et al. A prospective study of human immunodeficiency virus type 1 infection and the development of AIDS in subjects with hemophilia. $N$ Engl J Med. 1989;321(17):1141-1148.

2. Moss AR, Osmond D, Bacchetti P, et al. Risk factors for AIDS and HIV seropositivity in homosexual men. Am $J$ Epidemiol. 1987;125(6):1035-1047.

3. Phillips AN, Lee CA, Elford J, et al. Serial CD4 lymphocyte counts and development of AIDS. Lancet. 1991;337(8738):389-392.

4. Phillips AN, Elford J, Sabin C, et al. Immunodeficiency and the risk of death in HIV infection. JAMA. 1992;268(19):2662-2666.

5. Phillips AN, Lee CA, Elford J, et al. The cumulative risk of AIDS as the CD4 lymphocyte count declines. J Acquir Immune Defic Syndr. 1992;5:148-152.

6. Cao Y, Qin L, Zhang L, et al. Virologic and immunologic characterization of long-term survivors of human immunodeficiency virus type 1 infection. N Engl J Med. 1995;332(4):201-208. 
7. Buchbinder SP, Mehrotra DV, Duerr A, et al. Efficacy assessment of a cell-mediated immunity HIV-1 vaccine (the Step Study): A doubleblind, randomised, placebo-controlled, test-of-concept trial. Lancet. 2008;372(9653):1881-1893.

8. Gail MH, Rosenberg PS, Goedert JJ. Therapy may explain recent deficits in AIDS incidence. J Acquir Immune Defic Syndr. 1990;3(4):296-306.

9. Saravolatz L, Collins, G, Hodges D, et al. A randomized comparative trial of ZDV versus ZDV plus ddl versus ZDV plus ddC in persons with
CD4 cell counts of $<2 \mathrm{OO} / \mathrm{mm} 3$. In Proceedings of the Third National Conference on Retroviruses and Opportunistic Infections, Washington, USA.v1996.

10. Burcham J, Marmor M, Dubin N, et al. CD4\% is the best predictor of development of AIDS in a cohort of HIV-infected homosexual men. AIDS. 1991;5:365-372. 